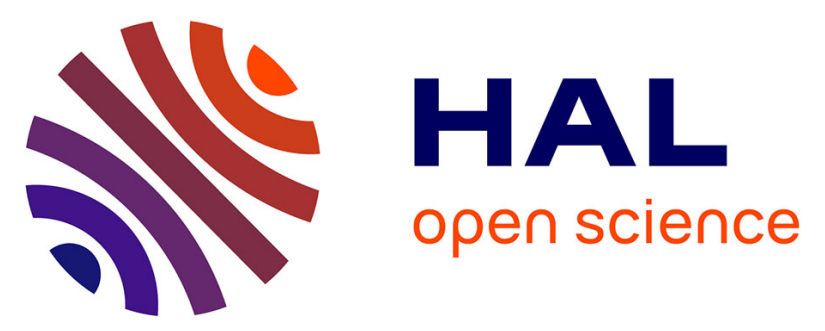

\title{
Towards an alternative to nano-QSAR for nanoparticle toxicity ranking in case of small datasets
}

Valérie Forest, Jean-François Hochepied, Lara Leclerc, Adeline Trouvé, Khalil Abdelkebir, Gwendoline Sarry, Vincent Augusto, Jérémie Pourchez

\section{- To cite this version:}

Valérie Forest, Jean-François Hochepied, Lara Leclerc, Adeline Trouvé, Khalil Abdelkebir, et al.. Towards an alternative to nano-QSAR for nanoparticle toxicity ranking in case of small datasets. Journal of Nanoparticle Research, 2019, 21 (5), 10.1007/s11051-019-4541-2 . hal-02132406

\section{HAL Id: hal-02132406 https://hal.science/hal-02132406}

Submitted on 17 May 2019

HAL is a multi-disciplinary open access archive for the deposit and dissemination of scientific research documents, whether they are published or not. The documents may come from teaching and research institutions in France or abroad, or from public or private research centers.
L'archive ouverte pluridisciplinaire HAL, est destinée au dépôt et à la diffusion de documents scientifiques de niveau recherche, publiés ou non, émanant des établissements d'enseignement et de recherche français ou étrangers, des laboratoires publics ou privés. 
Towards an alternative to nano-QSAR for nanoparticle toxicity ranking in case of small datasets

Valérie Forest*a, Jean-François Hochepied ${ }^{\mathrm{b}, \mathrm{c}}$, Lara Leclerc ${ }^{\mathrm{a}}$, Adeline Trouvée $^{\mathrm{b}, \mathrm{c}}$, Khalil Abdelkebir $^{\mathrm{b}, \mathrm{c}}$, Gwendoline Sarry $^{\mathrm{a}}$, Vincent Augusto $^{\mathrm{d}}$, Jérémie Pourchez ${ }^{\mathrm{a}}$.

${ }^{\text {a } M i n e s ~ S a i n t-E t i e n n e, ~ U n i v ~ L y o n, ~ U n i v ~ J e a n ~ M o n n e t, ~ I N S E R M, ~ U ~} 1059$ Sainbiose, Centre CIS, F-42023 Saint-Etienne, France.

${ }^{\mathrm{b}}$ Mines ParisTech, PSL Research University, MAT - Centre des matériaux, CNRS UMR 7633, BP 8791003 Evry, France.

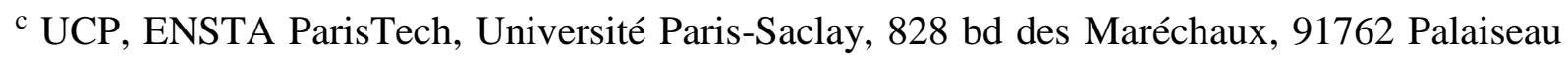
cedex France.

${ }^{\mathrm{d}}$ Mines Saint-Etienne, Univ Clermont Auvergne, CNRS, UMR 6158 LIMOS, Centre CIS, F42023 Saint-Etienne France.

* Corresponding author:

Valérie Forest:

Mines Saint-Etienne,

158 cours Fauriel, CS 62362, 42023 Saint-Etienne Cedex 2. FRANCE.

Email address: vforest@emse.fr - Telephone number: +33477499776

ORCID: 0000-0003-3124-0413

\section{Acknowledgements:}

The authors would like to acknowledge the funding from Institut Mines Telecom, ROQSTAR grant. 


\begin{abstract}
Statistical analysis approaches have been developed to predict the biological response to nanoparticles, especially quantitative structure-activity relationship (QSAR) models. But one major limitation remains the quantitative lack of data to build accurate models. The aim of this study was to investigate if simple alternative mathematical models could rank nanoparticles in a very binary way (i.e. toxic or not) in case of small dataset. We synthesized and characterized 25 nanoparticles from 6 metal (hydr)oxide families with particle size and shape tuning. We assessed their toxicity on RAW 264.7 cells and investigated relationships with both physicochemical and dimensional descriptors. A simple partial least square (PLS) regression analysis allowed ranking nanoparticles with respect to their toxicity, without false-negative results. But this model was not predictive due to the specific response of each family to dimensional parameters variations. A classification tree extracted the same main bulk descriptor as PLS, but interestingly showed the relevance of dimensional descriptors for the second and third node. We thus recommend the development of family-specific models and propose the combination of these two simple methods as pre-screening tools, a compromise to bridge the gap between case-by-case studies (expensive and time-consuming) and sophisticated nano-QSAR models (not suitable for small datasets).
\end{abstract}

\title{
Key-words
}

Nanoparticles; Nanotoxicology; Ranking; Small datasets; Nanodescriptors. 


\section{Introduction}

During the last decades, nanotechnologies and their applications have amazingly expanded leading to a growing public concern about the toxicity of manufactured nanoparticles. The huge number and variety of engineered nanoparticles makes it impossible to test them on a case-bycase basis (Chatterjee 2009). In addition, this approach would require intensive animal testing to obtain for each nanoparticle the necessary data (Dekkers et al. 2016). It appears therefore necessary to develop alternative approaches to help prioritize materials that need the most rigorous testing, at the same time prioritizing financial and human resources available in toxicological investigations (Chatterjee 2009; Puzyn et al. 2011; Gajewicz et al. 2012; Winkler et al. 2013).

To meet such expectations, predictive models are currently being developed. In particular, the Quantitative Structure-Activity Relationship (QSAR) model represents a promising approach. It was initially developed in the 1960's for chemical compounds (Hansch et al. 1962). As an example, QSAR approach is commonly used for risk assessment of pesticides. Indeed, due to a growing need for toxicity assessment and the increasing variety and number of products, regulatory agencies and legislations, such as REACH (Registration, Evaluation and Authorisation of Chemical, the European legislation on chemicals) encourage the use of alternatives to animal testing. Computational approaches can accelerate advances in (eco)toxicological understanding as they support the experimental data with additional in silico studies and results (Mas et al. 2010; Hamadache et al. 2017; Villaverde et al. 2017, 2018). It was recently proposed to transpose QSAR models to nanomaterials to rapidly and cheaply screen and predict their toxicity (Pan et al. 2016). This adaptation is still at the development stage and is not without challenges (Gajewicz et al. 2012). To continue with the example of pesticides, the environmental fate and behavior of these products formulated as nano are 
complex and vary from traditional formulations, arguing for the need of innovative and adapted risk assessment methodologies (Villaverde et al. 2018).

But the usefulness of computational approach in the nano field is constantly rising as illustrated by some interesting results reported in the literature. In particular, Puzyn et al. (Puzyn et al. 2011; Kar et al. 2014) have recently applied "nano-QSAR" starting from a dataset grouping 17 metallic oxide particles all differing by their chemical composition, and concluded that their toxicity to Escherichia coli bacteria could be predicted on the basis of the chemical nature only thanks to calculated or experimental descriptors such as metal electronegativity and cation charge. Surprisingly, dimensional or shape descriptors did not play any role. Gajewicz et al. (2015) confirmed these observations in a model of mammalian cells, the HaCaT cell line, regarding the toxicity of 18 metal oxide nanoparticles. More recently, Pan et al. (2016) demonstrated that in addition to the cationic charge as shown by Puzyn et al., the individual size and aggregation size of metal oxides nanoparticles were important factors for the cytotoxicity to both E. coli and HaCaT cells.

Despite these promising results, some hurdles remain to overcome. They may be roughly classified into 3 categories: i) the choice of the most suitable nanodescriptors, ii) the choice of the most relevant biological assays; and last but not least, iii) the quality and quantity of the data. Firstly, regarding the choice of the physico-chemical descriptors, many have been proposed as suitable for nanomaterials, they can be either derived from experimental data (for instance dimensional descriptors) or calculated with various theoretical approaches (Winkler et al. 2013; Gajewicz et al. 2015b; Pan et al. 2016). Often, physico-chemical descriptors are calculated, not assessed experimentally, and they usually do not follow the entire list of parameters described in the ISO recommendations (ISO/TR 13014:2012). In addition, almost always, only one sample per chemical composition is used. Secondly, a successful nano-QSAR also requires well-selected biological assays, at the same time facing the specific issues and 
concern about nanoparticles and providing valuable data (biological descriptors) for the QSAR model (Fourches et al. 2010; Gajewicz et al. 2012; Dekkers et al. 2016). Thirdly, and most of all, the main issue of nano-QSAR lies in the quality and quantity of data. A sufficient number of samples (particles with well-defined and distinct physico-chemical descriptors) should be available to build a statistically acceptable dataset (Fourches et al. 2010; Dekkers et al. 2016). Indeed, traditional QSAR approach is based on very large datasets (hundreds or even thousands substances). However, available experimental data for nanoparticles are still not enough (units or tens, in the best case) and empirical data and physico-chemical and biological characterization are not systematic when they are not inconsistent (Gajewicz et al. 2012).

Thus, although nano-QSAR models have been the topic of intensive research during the last two decades their use is actually limited, mainly because of the small size of the dataset available for modeling. We may therefore wonder if alternative mathematical models, less complex and less sophisticated cannot be sufficient to rank, although more modestly but still efficiently, nanomaterials with respect to their toxicity in case of small datasets. And indeed, recently, other approaches have gained interest such as classification trees or read-across basedapproaches (Gajewicz 2017a, b; Gajewicz et al. 2018). In this context, the aim of the present study was to determine if tools as simple as a simple partial least square regression or decision trees could accurately rank nanoparticles (i.e. toxic $v$ s. non toxic) based on a small dataset. To compensate the quantitative lack of data, their quality must be optimal. We therefore synthesized 25 nanoparticles from 6 metal (hydr)oxide families we then thouroughly characterized according to the ISO standards (ISO/TR 13014:2012). We next assessed their toxicity in vitro using a model of macrophages and biological endpoints used in empirical nanotoxicology studies. This is the main originality of this work along with the synthesis, systematical and empirical physico-chemical characterization of the nanoparticles. Based on 
these experimental data two predictive models and a decision tree were constructed and allowed us to discuss about the relevance of these approaches.

\section{Experimental}

\section{Nanoparticle synthesis}

25 nanoparticles from 6 metal (hydr)oxide families $\left(\mathrm{SiO}_{2}, \mathrm{TiO}_{2}, \mathrm{CeO}_{2}, \mathrm{AlOOH}, \mathrm{ZnO}, \mathrm{Ni}(\mathrm{OH})_{2}\right)$ were synthesized. In each chemical family particle size and shape were tuned to ensure an independent analysis of chemical and dimensional descriptors.

The synthesis of boehmite particles was inspired from Yang (2011). Boehmite particles were synthesized by room temperature precipitation of aluminium chloride with soda-ammonia solutions followed by hydrothermal ripening at various $\mathrm{pH}$ values $(4.5,6$ and 8.5$)$ in autoclaves at $180^{\circ} \mathrm{C}$ during $24 \mathrm{~h}$ or microwave heating (Monowave 300 , Anton Paar) at $180^{\circ} \mathrm{C}$ during 10 minutes. The objective was to control shape from platelets to rods. All samples were washed by successive centrifugations in water, then freeze-dried. Table 1 summarises the experimental conditions. AlOOH-1 and AlOOH-3 were supposed to give the same particles but samples exhibited a different specific surface area and agglomeration level in bundles, which may be due to accidental temperature oscillation in the autoclave and/or $\mathrm{pH}$ shift before heating.

Table 1 - Experimental conditions for the synthesis of boehmite particles.

\begin{tabular}{|c|c|c|c|}
\hline Sample & pH & Heating device & Ripening \\
\hline AlOOH-1 & 4.5 & Autoclave & $24 \mathrm{~h}$ \\
\hline AlOOH-2 & 8.5 & Autoclave & $24 \mathrm{~h}$ \\
\hline AlOOH-3 & 4.5 & Autoclave & $24 \mathrm{~h}$ \\
\hline AlOOH-4 & 6 & Autoclave & $24 \mathrm{~h}$ \\
\hline AlOOH-5 & 6 & Microwave & $10 \mathrm{~min}$ \\
\hline
\end{tabular}

Synthesis of nickel hydroxide particles: nickel hydroxide nanoparticles are nanoplatelets, a shape induced by the brucite structure. For samples $\mathrm{Ni}(\mathrm{OH})_{2}-1$ to 4 , nickel hydroxide was precipitated in a vortex rapid mixer (whose geometry was described by Di Patrizio et al. (2016)) 
by mixing nickel nitrate $0.05 \mathrm{M}$ solution and soda-ammonia solutions. The control of particle characteristics (size, agglomeration) was obtained by tuning soda/ammonia ratio and concentrations as reported in Table $2 . \mathrm{Ni}(\mathrm{OH})_{2}-5$ particles were obtained by a protocol described by Coudun et al. (2005), allowing "stack of pancakes" morphology. All samples were washed by successive centrifugations in water, then freeze-dried. $\mathrm{NiO}$ sample was then obtained by calcining $\mathrm{Ni}(\mathrm{OH})_{2}-4$ at $300^{\circ} \mathrm{C}$ during $4 \mathrm{~h}$ under air.

Table 2 - Soda/ammonia ratios for the synthesis of nickel hydroxide particles.

\begin{tabular}{|c|c|}
\hline Sample & {$[\mathbf{N a O H}] /\left[\mathbf{N H}_{3}\right]$} \\
\hline $\mathrm{Ni}(\mathrm{OH})_{2}-1$ & $0.1 / 0.3$ \\
\hline $\mathrm{Ni}(\mathrm{OH})_{2}-2$ & $0.1 / 0$ \\
\hline $\mathrm{Ni}(\mathrm{OH})_{2}-3$ & $0.1 / 0.3^{*}$ \\
\hline $\mathrm{Ni}(\mathrm{OH})_{2}-4$ & $0.01 / 0.3$ \\
\hline
\end{tabular}

*For sample $\mathrm{Ni}(\mathrm{OH})_{2}-3$, ammonia was pre-mixed with nickel nitrate solution then the solution was mixed with soda in the rapid mixer.

The synthesis of zinc oxide particles (slightly elongated particles or short rods) was inspired from Trenque et al. (2013). Dihydrated zinc acetate was dissolved in diethyleneglycol (0.1 M for $\mathrm{ZnO}-1,1 \mathrm{M}$ for $\mathrm{ZnO}-2)$ then the solutions were heated at $200^{\circ} \mathrm{C}$ by microwave irradiation (Monowave 300) during 3 minutes.

The synthesis of cerium dioxide particles was inspired from Florea et al. (2013). Our protocols of precipitation of cerium(III) nitrate with soda-ammonia solution followed by hydrothermal ripening were previously described (Forest et al. 2017), they allowed shape tuning from isotropic particles to nanorods.

Synthesis of titanium dioxide nanoparticles: for $\mathrm{TiO}_{2}-1$, a $0.5 \mathrm{M}$ titanium butoxide and $1 \mathrm{M}$ triethanolamine solution was heated at $180^{\circ} \mathrm{C}$ during $10 \mathrm{~min}$ by microwave irradiation. The sample was washed by successive centrifugations in water then freeze-dried. $\mathrm{TiO}_{2}-2$ is an 
amorphous product resulting from titanium butoxide hydrolysis at room temperature. $\mathrm{TiO}_{2}-3$ (spheres) and $\mathrm{TiO}_{2}-4$ (rods) were commercial products from Sigma-Aldrich.

The synthesis of silica nanoparticles was inspired from Music et al. (2011). A solution of sodium silicate $26.5 \%$ was diluted 4 times in water, heated at $80^{\circ} \mathrm{C}$ and acidified to $\mathrm{pH}=2$ by dropping a $1.8 \mathrm{M}$ sulfuric acid solution. For $\mathrm{SiO}_{2}-1$ heating was maintained during 45 min after acidification without $\mathrm{pH}$ control, for $\mathrm{SiO}_{2}-2$ heating was maintained during 90 min and $\mathrm{pH}$ value was maintained at 2 by sulfuric acid addition during heating. The samples were washed by successive centrifugations in water.

\section{Nanoparticle physico-chemical characterization}

Following ISO guidelines (ISO/TR 13014:2012), the following physico-chemical descriptors were considered:

- nanoparticle size: average dimensions were measured by Transmission Electron Microscopy (TEM) using a FEI TECNAI 20FST operating at $200 \mathrm{kV}$. Each particle was defined by 1 (sphere) or 2 (rods/plates) dimensions (sufficient considering that a revolution axis practically always exists): dmax and dmin.

- chemical composition: known from synthesis conditions and X-ray diffraction. Associated descriptors were $\mathrm{x}\left(\mathrm{H}_{2} \mathrm{O}\right)$ from the raw formula $\mathrm{M}(\mathrm{n}+) \mathrm{O}(\mathrm{n} / 2) \cdot \mathrm{xH}_{2} \mathrm{O}$, the oxidation degree of the metal (cation charge) n_oxy_M, the radius of the metallic cation $\mathrm{r}_{-}$cat, and the Pauling electronegativity of the metallic element EN_M. We decided to introduce $x\left(\mathrm{H}_{2} \mathrm{O}\right)$ to make a chemical difference (in addition to solubility) between oxides and hydroxides, the other 3 descriptors were chosen from their occurrence in literature.

- solubility: we considered the solubility of the bulk materials at $\mathrm{pH}=7$, from tables.

- shape: shape factor was classically defined as $\mathrm{SF}=\mathrm{dmax} / \mathrm{dmin}$ from dimensions by TEM, and we also introduced a corrected shape factor to discriminate between rods and plates, which is 
of course critical since rods have 2 dimensions in the nano-range when plates only have 1: considering the length of the axis of revolution $\left(\mathrm{d}_{\mathrm{axis}}\right)$ and the dimension perpendicular to this axis $\left(d_{\text {perp }}\right)$, the corrected shape factor was defined as CSF $=\log \left(d_{\text {axis }} / d_{\text {perp }}\right)$. This way, we have different domains of CSF for each shape: for rods $\mathrm{CSF}>0$, spheres $\mathrm{CSF}=0$, plates $\mathrm{CSF}<0$.

- the zeta potential was measured at $\mathrm{pH}=7$ by dispersing nanoparticles in water by ultrasonic treatment and using a nanoZS (Malvern Instrument, UK).

- the specific surface area SSA was measured on dried powders by nitrogen gas adsorption at 77K (BET method) with an ASAP2000 instrument from Micromeritics.

- the crystalline structure was determined by X-ray diffraction using a Bruker D8 diffractometer in $\theta-\theta$ geometry and Co $K_{\alpha 1}$ radiation $(\lambda=1.789 \AA)$ to check there was no minor phase in our syntheses, but was not introduced as a descriptor.

- the agglomeration/aggregation state: agglomeration is difficult to quantify and there is no associated established metrology, especially when agglomeration (weak forces between particles) and aggregation (strong forces or bridges) may both occur and evolve during biological tests. To keep it simple, we just defined 3 classes from TEM observations and stability/dispersibility when handling suspensions: $\mathrm{Agg}=0$ : well-dispersed particles, $1=$ small (submicron) or fluffy agglomerates, $2=$ dense or big (micrometric) agglomerates and likely aggregates.

\section{Cellular model}

The RAW 264.7 cell line derived from mice peritoneal macrophages transformed by the Abelson murine leukemia virus and was provided by ATCC Cell Biology Collection (Promochem, LGC, Molsheim, France). Cells were cultured in Dulbecco Modified Eagle Medium (DMEM) complemented with $10 \%$ of fetal calf serum and $1 \%$ of penicillinstreptomycin (called DMEMc) at $37^{\circ} \mathrm{C}$ under a 5\% carbon dioxide humidified atmosphere. 
Cells were seeded in 96-well-plates (100 000 cells in $50 \mu \mathrm{L}$ of medium per well) and were allowed to adhere overnight. Nanoparticles were diluted in cell culture medium to reach the following final concentrations: $15,30,60$ and $120 \mu \mathrm{g} / \mathrm{mL}$. Nanoparticles were added to cells and further incubated for $24 \mathrm{~h}$.

\section{Empirical toxicity testing: the lactate dehydrogenase $(\mathrm{LDH})$ release}

To evaluate cell membrane integrity, the cellular release in the supernatant of cytoplasmic lactate dehydrogenase was assessed using the CytoTox-96 ${ }^{\mathrm{TM}}$ Homogeneous Membrane Integrity Assay (Promega, Charbonnières-les-Bains, France) according to the manufacturer's instructions. The optical density of the samples was determined using a microplate reader (Multiskan RC; Thermolabsystems, Helsinki, Finland) set to $450 \mathrm{~nm}$. Three independent experiments were performed, each in quadruplicate and the activity of the released LDH was reported to that of negative control cells (incubated without nanoparticles). A positive control consisted in the maximal cellular LDH released after cells lysis.

\section{Expression of the toxicity results: biological scores}

Regarding the biological assays, three independent experiments were performed in quadruplicate. Means were calculated between the 4 doses to get an average signal and this signal was normalized to the negative control (signal obtained for the cells incubated alone, i.e. without nanoparticles). Therefore, the basal signal obtained for the control was set to 1 . As explained further, a threshold of 1.5 was selected to rank particles, i.e. a nanoparticle with a score $>1.5$ was considered as cytotoxic.

\section{Partial least square regression analysis}


The physicochemical descriptors included in this study were selected either because they were recommended by the ISO/TR 13014:2012 guideline or because they have been reported in the literature to be predictive of nanoparticle toxicity. A partial least square regression (PLS) analysis was performed using $\mathrm{R}$ software to build and compare two models. The fisrt one included the 4 composition-related "non nano" (non dimensional) descriptors and the second one considered all (12) descriptors thus mixing chemical and dimensional descriptors. The root mean square error of prediction (RMSEP) versus the number of components was used to select the best number of components (dimensions) and the coefficients of the linear equation were validated by a leave-one-out (LOO) approach. Considering the relatively low number of samples and our main objective (to predict a binary toxic/non toxic behavior considering both physico-chemical and dimensional descriptors), we prefered the LOO approach to methods based on training and validation sets, more relevant to bigger datasets. The $\mathrm{R}^{2}$ was calculated to estimate the goodness of fit of our models (i.e. the ability to reproduce the data in the training set). For internal validation we used the leave-one-out-cross-validation (LOO-CV, especially recommended for small datasets (Gramatica 2007)) and calculated the $\mathrm{Q}^{2} \mathrm{LOO}$ to evaluate the robustness and predictivity of our models. Finally, as our dataset is composed of (hydr)oxide nanoparticles, the applicability domain of the models is defined as such.

\section{Decision tree}

Decision tree classification is a classic classification method in which records are labeled and classified into different levels of a response variable according to the frequency with which a set of features appear when a certain level of the response variable takes place. The algorithm implemented in this method is known as CART (Classification And Regression Tree). The main goal of the CART algorithm is to produce a classification tree - a set of classifier branches that determines the set of rules or variable interactions capable of predicting a given outcome 
(response variable). The objective in the construction of these classification rules is to make possible the prediction of the occurrence of such outcomes in future scenarios using the information from the set of variables and rules described by the decision tree (Kar et al. 2014). Considering the small size of the dataset, we applied the leave-one-out approach for crossvalidation. Each learning set is created by taking all the samples except one, the test set being the sample left out. Thus, for $n$ samples, we have $n$ different training sets and $n$ different tests set. This cross-validation procedure does not waste much data as only one sample is removed from the training set. We implemented all algorithms using the Python language and the SciKit learn library for machine learning methods. To assess the performance of the prediction, we provided the following metrics: accuracy, sensitivity, specificity and error rate:

$$
\begin{gathered}
\text { Accuracy }=(\mathrm{tp}+\mathrm{tn}) /(\mathrm{tp}+\mathrm{tn}+\mathrm{fp}+\mathrm{fn}) \\
\text { Sensitivity }=\mathrm{tp} /(\mathrm{tp}+\mathrm{fn}) \\
\text { Specificity }=\mathrm{tn} /(\mathrm{tn}+\mathrm{fp}) \\
\text { Error rate }=\mathrm{fn} /(\mathrm{fn}+\mathrm{tp})
\end{gathered}
$$

where tp, fp, tn and fn denote the number of true positives, false positives, true negatives and false negatives respectively.

Finally, in order to provide a decision aid tool to predict the toxicity of any new compound, we proposed an innovative approach to build an average tree, representing the best consensus of the $n$ decision trees.

\section{Results}

Our approach considered, in the family of metallic oxides and hydroxides, 25 nanoparticles of 6 chemical compositions with at least 2 significantly different particles (in size and/or shape) in each chemical family to ensure an independent analysis of chemical and dimensional descriptors. Nanoparticle samples were characterized to quantify the relevant experimental 
descriptors. A toxicity test, commonly used in nanotoxicology, was performed: the assessment of the LDH release from RAW 264.7 cells. The results were analyzed by a home-made ranking method. Our aim was to predict a binary behavior (probably toxic versus probably non toxic) considering both physico-chemical and dimensional descriptors using a simple mathematical model. The idea was to propose a ranking tool for rapid and cheap pre-screening purpose. The biological scores were determined for global ranking purposes with usual dose range of nanoparticles used in in vitro nanotoxicology studies and did not directly take into account the impact of the nanoparticle dose.

Following ISO guidelines (ISO/TR 13014:2012), the following physico-chemical descriptors were considered: nanoparticle size, chemical composition with associated descriptors (such as the hydration rate, the oxidation degree of the metal (cation charge), the radius of the metallic cation, and the Pauling electronegativity of the metallic element), solubility, shape, the zeta potential, the specific surface area SSA, the crystalline structure, and the agglomeration/aggregation state. Table 3 details the nanoparticle descriptors as well as the biological endpoint (score from the LDH assay) forming the dataset used in this study. 
Table 3 - Dataset used for the construction of the models.

\begin{tabular}{|c|c|c|c|c|c|c|c|c|c|c|c|c|c|}
\hline Sample & $\begin{array}{l}\text { Hydratio } \\
\text { n rate } \\
x(\mathrm{H} 2 \mathrm{O})\end{array}$ & $\begin{array}{l}\text { Oxidation } \\
\text { degree of } \\
\text { the metal } \\
\text { n_oxy_M }\end{array}$ & $\begin{array}{c}\text { Radius of } \\
\text { the metallic } \\
\text { cation } \\
\text { r_cat }\end{array}$ & $\begin{array}{c}\text { Pauling } \\
\text { electronegativity } \\
\text { EN_M }\end{array}$ & $\begin{array}{l}\text { Solubility } \\
\text { s(log Mtot })\end{array}$ & $\begin{array}{c}\text { Zeta } \\
\text { potential } \\
\mathrm{pH}=7 \\
\text { zeta }\end{array}$ & $\begin{array}{l}\text { Specific } \\
\text { surface } \\
\text { area } \\
\text { SSA }\end{array}$ & dmin & sions & $\begin{array}{c}\text { Shape factor } \\
=\mathrm{dmax} / \mathrm{dmin} \\
\mathrm{SF}\end{array}$ & $\begin{array}{c}\text { Corrected shape } \\
\text { factor=log(daxis/ } \\
\text { dperp) } \\
\text { CSF }\end{array}$ & $\begin{array}{l}\text { Agglomeration/ } \\
\text { aggregation } \\
\text { state } \\
\text { Agg }\end{array}$ & LDH \\
\hline AlOOH-1 & 0.5 & 3 & 67.5 & 1.61 & -6.5 & 37 & 121 & 10 & 300 & 30 & 1.48 & 0 & 1.1 \\
\hline $\mathrm{AlOOH}-2$ & 0.5 & 3 & 67.5 & 1.61 & -6.5 & 39 & 107 & 10 & 30 & 3 & -0.48 & 0 & 1.08 \\
\hline AlOOH-3 & 0.5 & 3 & 67.5 & 1.61 & -6.5 & 37 & 62 & 6 & 300 & 50 & 1.7 & 1 & 1.05 \\
\hline $\mathrm{AlOOH}-4$ & 0.5 & 3 & 67.5 & 1.61 & -6.5 & 33 & 40 & 10 & 100 & 10 & 1 & 0 & 1.07 \\
\hline AlOOH-5 & 0.5 & 3 & 67.5 & 1.61 & -6.5 & 37 & 54 & 5 & 50 & 10 & 1 & 2 & 1.07 \\
\hline $\mathrm{Ni}(\mathrm{OH}) 2-1$ & 1 & 2 & 83 & 1.91 & -3.5 & 40 & 190 & 3 & 10 & 3 & -0.48 & 1 & 2.76 \\
\hline $\mathrm{Ni}(\mathrm{OH}) 2-2$ & 1 & 2 & 83 & 1.91 & -3.5 & 44 & 226 & 2 & 10 & 5 & -0.7 & 2 & 2.57 \\
\hline $\mathrm{Ni}(\mathrm{OH}) 2-3$ & 1 & 2 & 83 & 1.91 & -3.5 & 31 & 192 & 3 & 30 & 10 & -1 & 1 & 2.99 \\
\hline $\mathrm{Ni}(\mathrm{OH}) 2-4$ & 1 & 2 & 83 & 1.91 & -3.5 & 31 & 86 & 10 & 60 & 6 & -0.78 & 0 & 2.2 \\
\hline $\mathrm{Ni}(\mathrm{OH}) 2-5$ & 1 & 2 & 83 & 1.91 & -3.5 & 35 & 10 & 50 & 200 & 4 & -0.6 & 0 & 2.07 \\
\hline $\mathrm{NiO}$ & 0 & 2 & 83 & 1.91 & -12 & 35 & 40 & 10 & 30 & 3 & -0.48 & 0 & 0.88 \\
\hline $\mathrm{ZnO}-1$ & 0 & 2 & 88 & 1.65 & -2.5 & 15 & 93 & 10 & 10 & 1 & 0 & 2 & 2.62 \\
\hline $\mathrm{ZnO}-2$ & 0 & 2 & 88 & 1.65 & -2.5 & 29 & 19 & 40 & 150 & 4 & 0.6 & 1 & 2.81 \\
\hline $\mathrm{CeO} 2-1$ & 0 & 4 & 101 & 1.12 & -9.5 & -26 & 151 & 4 & 40 & 10 & 1 & 1 & 2.02 \\
\hline $\mathrm{CeO} 2-2$ & 0 & 4 & 101 & 1.12 & -9.5 & -30 & 52 & 5 & 5 & 1 & 0 & 2 & 2.58 \\
\hline $\mathrm{CeO} 2-3$ & 0 & 4 & 101 & 1.12 & -9.5 & -35 & 61 & 8 & 40 & 5 & 0.7 & 1 & 2.08 \\
\hline $\mathrm{CeO} 2-4$ & 0 & 4 & 101 & 1.12 & -9.5 & -17 & 60 & 18 & 18 & 1 & 0 & 1 & 0.98 \\
\hline $\mathrm{CeO} 2-5$ & 0 & 4 & 101 & 1.12 & -9.5 & -31 & 37 & 8 & 90 & 10 & 1 & 1 & 2.08 \\
\hline $\mathrm{CeO} 2-6$ & 0 & 4 & 101 & 1.12 & -9.5 & 9 & 61 & 15 & 15 & 1 & 0 & 1 & 0.82 \\
\hline TiO2-1 & 0 & 4 & 74.5 & 1.54 & -9 & 9 & 94 & 20 & 35 & 2 & 0.3 & 2 & 0.88 \\
\hline $\mathrm{TiO} 2-2$ & 0 & 4 & 74.5 & 1.54 & -9 & 1 & 302 & 2 & 2 & 1 & 0 & 2 & 1.01 \\
\hline TiO2-3 & 0 & 4 & 74.5 & 1.54 & -9 & -4 & 45 & 20 & 20 & 1 & 0 & 1 & 1.29 \\
\hline TiO2-4 & 0 & 4 & 74.5 & 1.54 & -9 & 0 & 44 & 10 & 50 & 5 & 0.7 & 2 & 1 \\
\hline
\end{tabular}


SiO2-1

SiO2-2
1.9

1.9
$-2.7$ $-2.7$
326

447
10
14 
A partial least square regression (PLS) analysis was performed. We first constructed a model with 4 chemical composition related descriptors (n_oxy_metal, xH2O, r_cation and EN-metal), thus excluding dimensional decriptors. Figure 1 plots the experimental LDH score versus the predicted score. Of course in this model all particles belonging to the same chemical family have the same predicted score, which is in general far from the experimental reality. According to RMSEP, a model with 2 components is appropriate and the obtained equation is:

Predicted $\mathrm{LDH}$ score $=0.560+0.153 \times\left(\mathrm{H}_{2} \mathrm{O}\right)-0.394$ n_oxy_metal +0.0263 r_cation +0.102 EN_metal.

Since these descriptors are not normalized we also give the order of the maximum variation of the score induced by each term: 0.15 for $\mathrm{x}\left(\mathrm{H}_{2} \mathrm{O}\right), 0.80$ for n_oxy_metal, 1.20 for $\mathrm{r} \_$cation and 0.08 for EN_metal. In other words, the cationic radius and the cation charge are the key descriptors, the introduction of $\mathrm{x}\left(\mathrm{H}_{2} \mathrm{O}\right)$ in addition to the 3 other descriptors used as a refinement to encompass all families from metallic hydroxides to oxides has a relatively low impact, even if not to be neglected, whereas EN_metal impact is very low.

$\mathrm{R}^{2}=0.718$ may be thought as a bit low to claim for a model, this is obviously due to the huge dispersion of experimental scores between samples with the same chemical composition, especially $\mathrm{CeO}_{2}$ and $\mathrm{Ni}(\mathrm{OH})_{2}$. This dispersion has nothing to do with poor data quality but is due to morphological and dimensional effects as shown in our previous study on ceria (Forest et al. 2017). Nevertheless, from a practical point of view, one must admit that this composition based "non dimensional" approach is sufficient to predict the non cytotoxicity of particles if we set a threshold value at score $=1.5$ (corresponding to the threshold above which the signal is considered significantly increased compared to the negative control, i.e. cells unexposed to nanoparticles), since no false negative results are obtained (all particles with experimental score $>1.5$ do have a predicted score $>1.5$ ). Only 3 particles are predicted as "cytotoxic" whereas they are not: 2 samples of $\mathrm{CeO}_{2}$ family, a material exhibiting a spectacular particle shape effect 
on nanotoxicity as we exhibited in a previous publication (Forest et al. 2017), and the $\mathrm{NiO}$ sample obtained by calcining one $\mathrm{Ni}(\mathrm{OH})_{2}$ sample, interesting as it shows that the correction brought by $\mathrm{x}(\mathrm{H} 2 \mathrm{O})$ is not totally sufficient. The point is the dispersion of experimental scores is low with "non toxic" families as $\mathrm{SiO}_{2}, \mathrm{TiO}_{2}$ and $\mathrm{AlOOH}$ whereas it is wide with cytotoxic families as $\mathrm{CeO}_{2}$ and $\mathrm{Ni}(\mathrm{OH})_{2}$. Clearly, our results call for introducing more $\mathrm{TiO}_{2}$ or $\mathrm{SiO}_{2}$ samples in future studies to check this assessment.

$\mathrm{Q}^{2}-\mathrm{LOO}=0.0$ is indeed very low and indicates a priori no predictability beyond our dataset, but one must also keep in mind that our approach is not to predict accurate scores but the right quadrant.

Then we tried to build a model mixing composition and dimensional descriptors. Considering now all the chosen descriptors, after a first model we observed that 3 descriptors could be discarded without damage: dmin due to its low effect, zeta potential and solubility at $\mathrm{pH} 7$ due to their strong correlations to other descriptors. Considering the remaining 9 descriptors, a model with 6 components $(\mathrm{RMSEP}=0.55)$ gives the coefficients reported in Table 4, with $\mathrm{R}^{2}=0.823$. Of course, due to the relatively low number of samples, there may be overfitting.

Table 4 - Descriptors chosen for the tentative second model and corresponding coefficients (not normalized).

\begin{tabular}{|c|c|c|c|c|c|c|c|c|c|c|}
\hline Variable & constant & x_H n_O $_{2}$ _oxy_M & r_cat & EN_M & SSA & dmax & SF & CSF & Agg \\
\hline coeff & -0.662 & 0.190 & -0.485 & 0.037 & 0.104 & 0.00193 & 0.00248 & -0.0111 & -0.0234 & 0.344 \\
\hline $\begin{array}{c}\text { variation range } \\
\text { of the term } \\
\text { variable*coeff }\end{array}$ & & 0.19 & 0.97 & 2.11 & 0.08 & 0.84 & 0.74 & 0.54 & 0.06 & 0.79 \\
\hline
\end{tabular}

Figure 2 plots the experimental versus the predicted LDH score with this second model. Compared to the previous model, the change of the coefficients of physico-chemical descriptors 
is around $20 \%$, and among the 5 dimensional descriptors, the corrected shape factor was found not significant whereas the relative weights of the 4 other ones were relatively close. Considering that the values quantifying the agglomeration/aggregation level were arbitrarily set to 0,1 or 2 depending on a very rough and qualitative approach, this point calls for more attention to develop appropriate metrological tools to quantify agglomeration and aggregation. As far as $\mathrm{Q}^{2}-\mathrm{LOO}$ is concerned, one may wonder that it is even worse than in the previous "model" and negative (!) $\mathrm{Q}^{2}-\mathrm{LOO}=-0.3$.

One may consider that the model is poor (if existing). This is obviously true but the real reason is a key result as we explain now. As seen by comparing both figures, the corrections coming from dimensional descriptors do improve the prediction for families as $\mathrm{Ni}(\mathrm{OH})_{2}, \mathrm{ZnO}, \mathrm{AlOOH}$ and $\mathrm{SiO}_{2}$ but not for $\mathrm{CeO}_{2}$ and even degrade the prediction for $\mathrm{TiO}_{2}$. The predicted cytotoxicity of $\mathrm{NiO}$ is significantly lowered but it remains a false positive. On the one hand, this full model has no practical benefit as compared to the simple previous model if we consider a classification based on a threshold at score=1.5. But on the other hand, it clearly shows that it does not work to mix composition and dimensional descriptors for a simple reason: the impact of dimensional descriptors does exist but is clearly family dependent. This is obvious considering for instance boehmite, where in spite of huge dimension and shape differences the experimental scores were more or less the same for all samples, whereas for ceria the shape variation induced a strong score variation. The same remarks would apply for all dimensional descriptors. At the end, the model does as best as possible to fit the results but there is no hope to be predictive this way. As a consequence, studies considering dimensional descriptors in order to predict toxicity make sense only if restricted to a given chemical family.

At the end of this PLS study, we observe that the prediction of the right quadrant is pretty good with only the cationic radius and the cation charge, and that dimensional parameters cannot help to improve the fate of the 3 false positive because we want to deal simultaneously with all 
families. This is a clear invitation to switch to decision tree, very suitable for our purpose and likely to extract more homogenous subgroups. Using the leave-one-out approach for cross validation, we computed 25 decision trees and reported the metrics to assess the quality of the prediction in Table 5.

Table 5 - Performance of LDH prediction. TP: true positive, TN: true negative, FP: false positive, FN: false negative.

\begin{tabular}{|c|c|c|c|c|}
\hline $\begin{array}{c}\text { Sub } \\
\text { sample }\end{array}$ & $\mathrm{TP}$ & TN & FP & $\mathrm{FN}$ \\
\hline 1 & & 1 & & \\
\hline 2 & & 1 & & \\
\hline 3 & & 1 & & \\
\hline 4 & & 1 & & \\
\hline 5 & & 1 & & \\
\hline 6 & & & 1 & \\
\hline 7 & 1 & & & \\
\hline 8 & 1 & & & \\
\hline 9 & 1 & & & \\
\hline 10 & 1 & & & \\
\hline 11 & & & & 1 \\
\hline 12 & & & 1 & \\
\hline 13 & 1 & & & \\
\hline 14 & 1 & & & \\
\hline 15 & & & 1 & \\
\hline 16 & 1 & & & \\
\hline 17 & & & & 1 \\
\hline 18 & 1 & & & \\
\hline 19 & & 1 & & \\
\hline 20 & & 1 & & \\
\hline 21 & & 1 & & \\
\hline 22 & & 1 & & \\
\hline 23 & & 1 & & \\
\hline 24 & & 1 & & \\
\hline 25 & & 1 & & \\
\hline Total & 8 & 12 & 3 & 2 \\
\hline Accuracy & 0.8 & & & \\
\hline Sensitivity & 0.8 & & & \\
\hline Specificity & 0.8 & & & \\
\hline Error rate & 0.2 & & & \\
\hline
\end{tabular}


For each subsample we reported the prediction result on the left out particle. The proposed method has a global accuracy of $80 \%$, a sensitivity of $80 \%$, a specificity of $80 \%$ and an error rate of $20 \%$. Although the prediction approach performs well, it would benefit further tests by extending the original dataset to other compounds.

The prediction model is improved using an average tree that is built using the 25 decision trees generated before. Figure 3 depicts the final average decision tree produced considering the common features included in the whole set of subsamples used to train the model. The decision tree branches include all rules and characteristics describing the different characteristics of nanoparticles. A test on the whole dataset has an accuracy, sensitivity and specificity of $100 \%$. The process to construct an average decision tree involved the identification of common features in all the 25 decision trees produced from the subsamples generated. Since all 25 decision trees share the same feature (metallic cation radius $<78.75$ ) at the root of the tree, it appeared that such feature was the most discriminatory criterion for the ranking of nanoparticles. SF criterion comes second for $72 \%$ of the generated trees, followed by dmax which is considered discriminant in $68 \%$ of the generated trees. Thus we are able to provide recommendations related to nanoparticles characteristics that should be examined first to decide on their toxicity, which are metallic cation radius, SF, and dmax.

Interestingly, the cation radius was the main descriptor according to PLS, whereas SF and dmax appeared as significant dimensional parameters in the second PLS model. So in spite of all the biases there is some consistency between both approaches and there was indeed an added value to keep them both in parallel.

\section{Discussion}


Models able to predict nanoparticle toxicity based on adequate descriptors are strongly needed. And as a matter of fact in silico models are the subject of intensive research and different computational models for nanomaterials have been reported (Lamon et al. 2018). Among them, QSAR models (Fourches et al. 2010; Puzyn et al. 2011; Winkler et al. 2013; Gajewicz et al. 2015b; Pan et al. 2016), read-across (Gajewicz et al. 2015a, 2017; Gajewicz 2017a, b), neural network (Fjodorova et al. 2017) or decision tree (Gajewicz et al. 2018) classifications. In the present study, we could not reasonably build a QSAR model because our dataset was too small. On the contrary, we chose PLS and decisions trees because of their simplicity and because they are easily accessible to a wider audience compared to other more complex computational methods. In this context, we showed that with a simple partial least square regression analysis it was possible to predict the cytotoxicity of a small dataset of (hydr)oxide metal nanoparticles and rank them without false-negative results considering only physico-chemical descriptors. Furthermore, a classification tree approach confirmed the high impact of chemical descriptors. Our results are perfectly consistent with data reported in the literature (Puzyn et al. 2011; Kar et al. 2014; Gajewicz et al. 2015b), that claimed a much higher impact of chemical descriptors as compared to dimensional descriptors to predict toxicity. But our approach was different as we did not exclude a priori size descriptors, we included them in our model. We evidenced there is undoubtedly a sharp impact of the particle shape with cerium dioxide (Forest et al. 2017). Since each chemical family has its own behavior, by mixing all families the correlation with dimensional descriptors is blurred. If we focus on only one family, chemical descriptors will be constant and the impact of dimensional descriptors will be correctly evaluated. But of course that means gathering many fully characterized samples differing by their size and shape for each chemical composition of practical interest.

One may regret that we used only one cell line but it has the advantage of being an eukaryote model, poorly described in the literature related to predictive models. Indeed, most existing 
studies have been carried out on prokaryotic systems (i.e. Escherichia coli bacteria). Regarding eukaryotic systems information is more scarce (Ghorbanzadeh et al. 2012; Pan et al. 2016). Another originality of the present work is the use of a biological assay commonly employed in standard nanotoxicology, but not so often when trying to build predictive models where more basic indicators (i.e. EC50) are usually used, being more or less informative. The LDH release reflects cytotoxicity and it appeared to be a relevant endpoint allowing classifying nanoparticles either in a toxic or in a safe category. We have tried to construct other models including two other parameters commonly used in standard nanotoxicology: the TNF- $\alpha$ production indicative of a pro-inflammatory response and the reactive oxygen species production signaling an oxidative stress (see Supplementary information). Suitable models could not be constructed, suggesting that the choice of the biological endpoints is not trivial and should be carefully considered, not all are relevant. Similarly, decision trees built with these data were not conclusive: when TNF- $\alpha$ production or reactive oxygen species production were tested as target variables, key performance indicators were not satisfying (data not shown). Last but not least, another asset of our study is the use of well characterized nanoparticles, with biological assays carried out in the same conditions contrary to studies where values are extracted from previously published studies and where data obtained from different sources are directly compared. This is a major issue as it is acknowledged that a fundamental limitation for any predictive model is the quality of the data used to build it (Gajewicz et al. 2012).

And indeed, the choice of descriptors is both crucial and delicate. Experimentally measured descriptors are rarely present and all the ISO standard parameters are not systematically integrated in predictive models described in the literature while they are mandatory to consider in any standard nanotoxicology study. An inappropriate choice of descriptors can be a source of bias in the results as we can only find correlations with initially included parameters while 
we can miss some important finding just because the suitable descriptors were not included $a$ priori.

Moreover, the idea of building family-specific predictive models could be a good compromise between the unsound search for a universal model able to predict the toxicity of all types of nanoparticles and the case-by-case study (that is too fastidious, highly time and moneyconsuming). Application of CART demonstrated good results for a specific target variable that is LDH release. It allowed to conclude on good predictive variables among physico-chemical characteristics of the nanoparticles. For further experiment, results provided using decisions trees can be used as a decision aid tool to decide on the most important features to measure experimentally. Also, the leave-one-out approach prevents the bias related to isolated training and test experiments and allows an objective validation of the provided decision aid tool as presented by Gajewicz et al. (2018). Decision tree classification appears as complementary to partial least square regression analysis as this latter allows a very binary classification of the nanoparticles (i.e. toxic or not toxic) while decision trees allow the identification of the main parameters involved in this toxicity. This could be of major interest, especially in the context of a "safer by design" approach.

\section{Conclusion}

The extension of traditional QSAR approach to nanoparticles faces some challenges, one of the biggest being the too small available datasets. With this study, based on the use of 25 thoroughly characterized metal oxide and hydroxide nanoparticles, we evidenced by partial least square regression analysis and decision tree classification relationships between toxicity and selected physical and chemical descriptors, able to rank nanoparticles in regard to their cytotoxicity without false-negative results. As illustrated by Figure 4, we thus propose these simple methods as pre-screening tools, a compromise able to bridge the gap between case-by-case studies (too 
fastidious, expensive and time-consuming) and more sophisticated QSAR models (efficient but not suitable for small datasets). These mathematical tools are very simple and can be easily extended to other types of nanoparticles, allowing to get new insights in the nano(eco)toxicology field.

\section{Conflict of Interest}

The authors declare that they have no conflict of interest.

\section{References}

Chatterjee R (2009) Calculating the costs of nanohazard testing. Environ Sci Technol 43:34053405. doi: 10.1021/es900758w

Coudun C, Hochepied J-F (2005) Nickel hydroxide "stacks of pancakes" obtained by the coupled effect of ammonia and template agent. J Phys Chem B 109:6069-6074. doi: 10.1021/jp0466441

Dekkers S, Oomen AG, Bleeker EAJ, et al (2016) Towards a nanospecific approach for risk assessment. Regul Toxicol Pharmacol RTP 80:46-59. doi: 10.1016/j.yrtph.2016.05.037

Di Patrizio N, Bagnaro M, Gaunand A, et al (2016) Hydrodynamics and mixing performance of Hartridge Roughton mixers: Influence of the mixing chamber design. Chem Eng J 283:375-387. doi: 10.1016/j.cej.2015.06.116

Fjodorova N, Novic M, Gajewicz A, Rasulev B (2017) The way to cover prediction for cytotoxicity for all existing nano-sized metal oxides by using neural network method. Nanotoxicology 11:475-483. doi: 10.1080/17435390.2017.1310949 
Florea I, Feral-Martin C, Majimel J, et al (2013) Three-Dimensional Tomographic Analyses of CeO2 Nanoparticles. Cryst Growth Des 13:1110-1121. doi: 10.1021/cg301445h

Forest V, Leclerc L, Hochepied J-F, et al (2017) Impact of cerium oxide nanoparticles shape on their in vitro cellular toxicity. Toxicol In Vitro 38:136-141. doi: 10.1016/j.tiv.2016.09.022

Fourches D, Pu D, Tassa C, et al (2010) Quantitative nanostructure-activity relationship modeling. ACS Nano 4:5703-5712. doi: 10.1021/nn1013484

Gajewicz A (2017a) Development of valuable predictive read-across models based on "reallife" (sparse) nanotoxicity data. Environ Sci Nano 4:1389-1403. doi: 10.1039/C7EN00102A

Gajewicz A (2017b) What if the number of nanotoxicity data is too small for developing predictive Nano-QSAR models? An alternative read-across based approach for filling data gaps. Nanoscale 9:8435-8448. doi: 10.1039/c7nr02211e

Gajewicz A, Cronin MTD, Rasulev B, et al (2015a) Novel approach for efficient predictions properties of large pool of nanomaterials based on limited set of species: nano-readacross. Nanotechnology 26:015701. doi: 10.1088/0957-4484/26/1/015701

Gajewicz A, Jagiello K, Cronin MTD, et al (2017) Addressing a bottle neck for regulation of nanomaterials: quantitative read-across (Nano-QRA) algorithm for cases when only limited data is available. Environ Sci Nano 4:346-358. doi: 10.1039/C6EN00399K

Gajewicz A, Puzyn T, Odziomek K, et al (2018) Decision tree models to classify nanomaterials according to the DF4nanoGrouping scheme. Nanotoxicology 12:1-17. doi: $10.1080 / 17435390.2017 .1415388$ 
Gajewicz A, Rasulev B, Dinadayalane TC, et al (2012) Advancing risk assessment of engineered nanomaterials: application of computational approaches. Adv Drug Deliv Rev 64:1663-1693. doi: 10.1016/j.addr.2012.05.014

Gajewicz A, Schaeublin N, Rasulev B, et al (2015b) Towards understanding mechanisms governing cytotoxicity of metal oxides nanoparticles: hints from nano-QSAR studies. Nanotoxicology 9:313-325. doi: 10.3109/17435390.2014.930195

Ghorbanzadeh M, Fatemi MH, Karimpour M (2012) Modeling the Cellular Uptake of Magnetofluorescent Nanoparticles in Pancreatic Cancer Cells: A Quantitative Structure Activity Relationship Study. Ind Eng Chem Res 51:10712-10718. doi: $10.1021 /$ ie3006947

Gramatica P (2007) Principles of QSAR models validation: internal and external. QSAR Comb Sci 26:694-701. doi: 10.1002/qsar.200610151

Hamadache M, Amrane A, Benkortbi O, et al (2017) Environmental Toxicity of Pesticides, and Its Modeling by QSAR Approaches. In: Roy K (ed) Advances in QSAR Modeling: Applications in Pharmaceutical, Chemical, Food, Agricultural and Environmental Sciences. Springer International Publishing, Cham, pp 471-501

Hansch C, Maloney PP, Fujita T, Muir RM (1962) Correlation of Biological Activity of Phenoxyacetic Acids with Hammett Substituent Constants and Partition Coefficients. Nature 194:178-180. doi: 10.1038/194178b0

ISO/TR 13014:2012 Nanotechnologies - Guidance on physico-chemical characterization of engineered nanoscale materials for toxicologic assessment. In: ISO. http://www.iso.org/iso/catalogue_detail?csnumber=52334. Accessed 28 Nov 2016 
Kar S, Gajewicz A, Puzyn T, et al (2014) Periodic table-based descriptors to encode cytotoxicity profile of metal oxide nanoparticles: a mechanistic QSTR approach. Ecotoxicol Environ Saf 107:162-169. doi: 10.1016/j.ecoenv.2014.05.026

Lamon L, Asturiol D, Vilchez A, et al (2018) Computational models for the assessment of manufactured nanomaterials: Development of model reporting standards and mapping of the model landscape. Comput Toxicol. doi: 10.1016/j.comtox.2018.12.002

Mas S, de Juan A, Tauler R, et al (2010) Application of chemometric methods to environmental analysis of organic pollutants: A review. Talanta 80:1052-1067. doi: 10.1016/j.talanta.2009.09.044

Musić S, Filipović-Vinceković N, Sekovanić L (2011) Precipitation of amorphous SiO2 particles and their properties. Braz J Chem Eng 28:89-94. doi: 10.1590/S010466322011000100011

Pan Y, Li T, Cheng J, et al (2016) Nano-QSAR modeling for predicting the cytotoxicity of metal oxide nanoparticles using novel descriptors. RSC Adv 6:25766-25775. doi: 10.1039/C6RA01298A

Puzyn T, Rasulev B, Gajewicz A, et al (2011) Using nano-QSAR to predict the cytotoxicity of metal oxide nanoparticles. Nat Nanotechnol 6:175-178. doi: 10.1038/nnano.2011.10

Trenque I, Mornet S, Duguet E, Gaudon M (2013) New insights into crystallite size and cell parameters correlation for $\mathrm{ZnO}$ nanoparticles obtained from polyol-mediated synthesis. Inorg Chem 52:12811-12817. doi: 10.1021/ic402152f 
Villaverde JJ, Sevilla-Morán B, López-Goti C, et al (2017) Computational Methodologies for the Risk Assessment of Pesticides in the European Union. J Agric Food Chem 65:20172018. doi: 10.1021/acs.jafc.7b00516

Villaverde JJ, Sevilla-Morán B, López-Goti C, et al (2018) Considerations of nanoQSAR/QSPR models for nanopesticide risk assessment within the European legislative framework. Sci Total Environ 634:1530-1539. doi: 10.1016/j.scitotenv.2018.04.033

Winkler DA, Mombelli E, Pietroiusti A, et al (2013) Applying quantitative structure-activity relationship approaches to nanotoxicology: current status and future potential. Toxicology 313:15-23. doi: 10.1016/j.tox.2012.11.005

Yang Q (2011) Synthesis of $\gamma$-Al2O3 nanowires through a boehmite precursor route. Bull Mater Sci 34:239-244. doi: 10.1007/s12034-011-0062-Z 


\section{Figure captions}

Fig. 1 Plot of experimentally determined versus predicted LDH scores with model 1

Fig. 2 Plot of experimentally determined versus predicted LDH scores with model 2

Fig. 3 Classification tree for the nanoparticle cytotoxicity. Cytotoxic nanoparticles are indicated in red, non-cytotoxic materials in green. The classification tree uses the auto-scaled values of

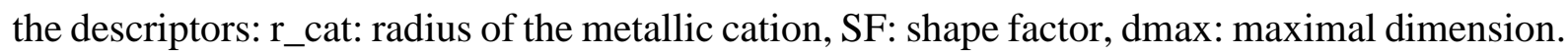
The Gini index represents a measure of statistical dispersion

Fig. 4 Partial least square regression and decision trees proposed as alternative approaches to case-by-case studies and QSAR models for the ranking of nanoparticle toxicity in case of small dataset. The advantages and drawbacks of each approach are summarized 
Figure 1

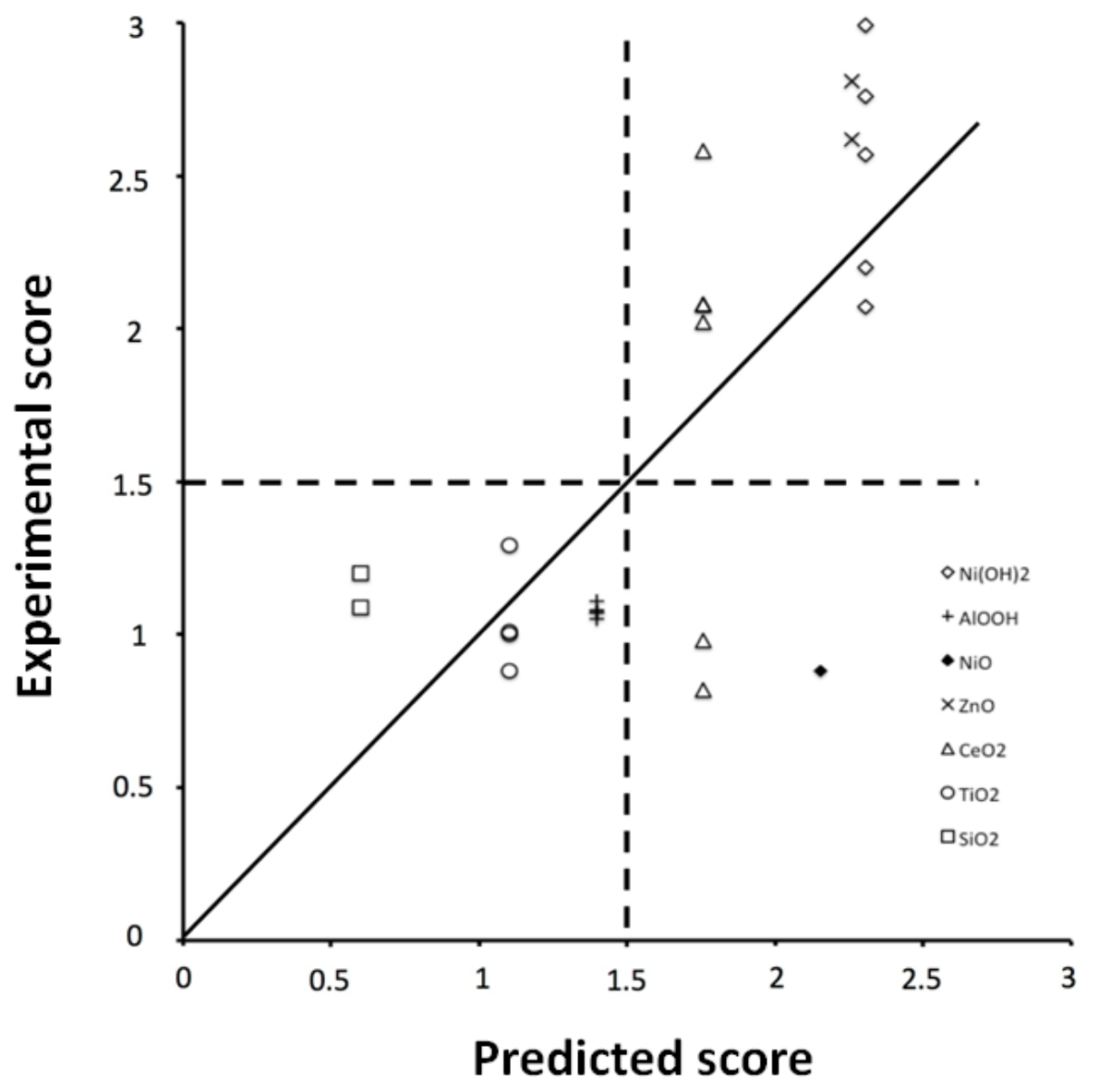


Figure 2

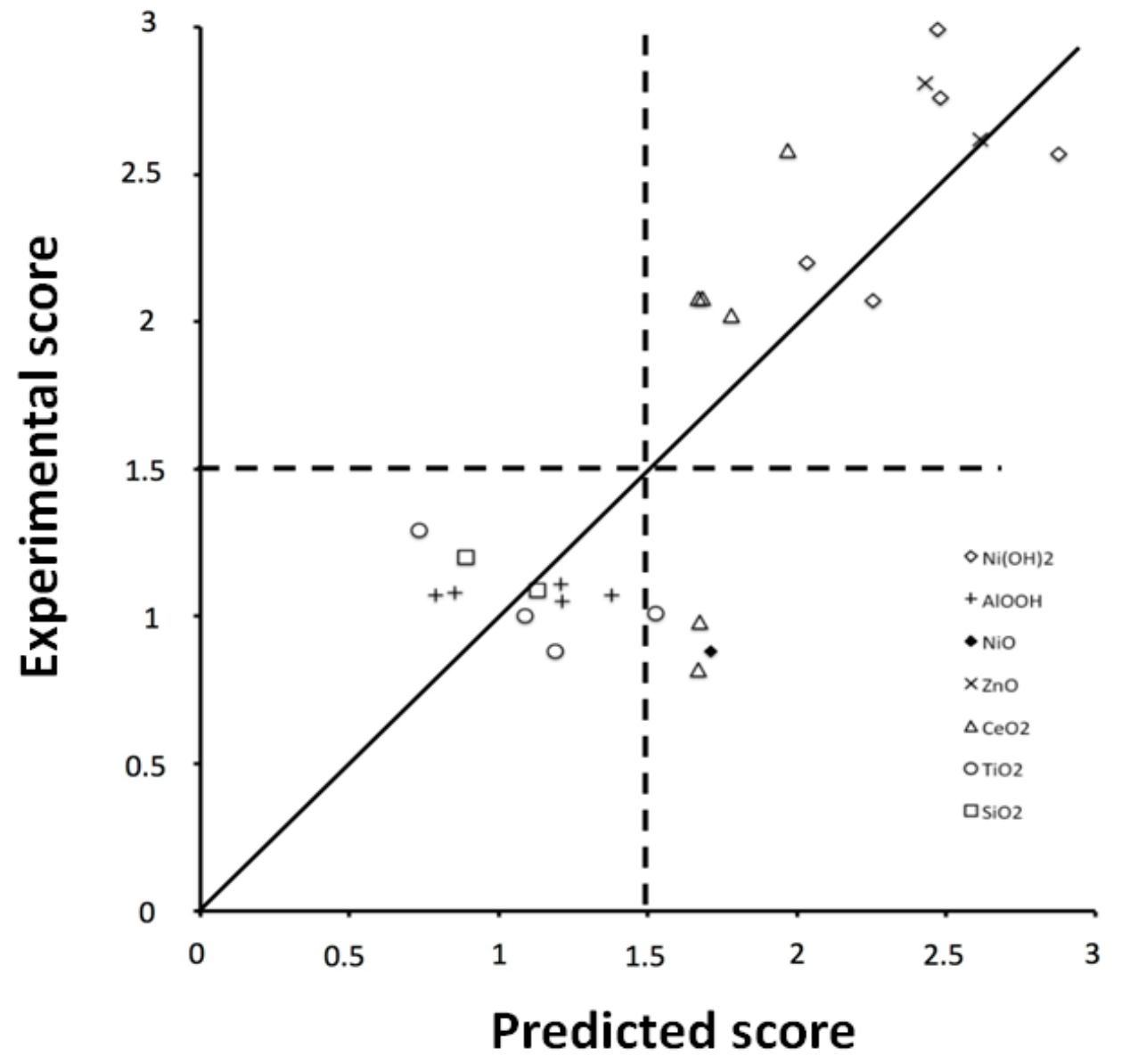




\section{Figure 3}

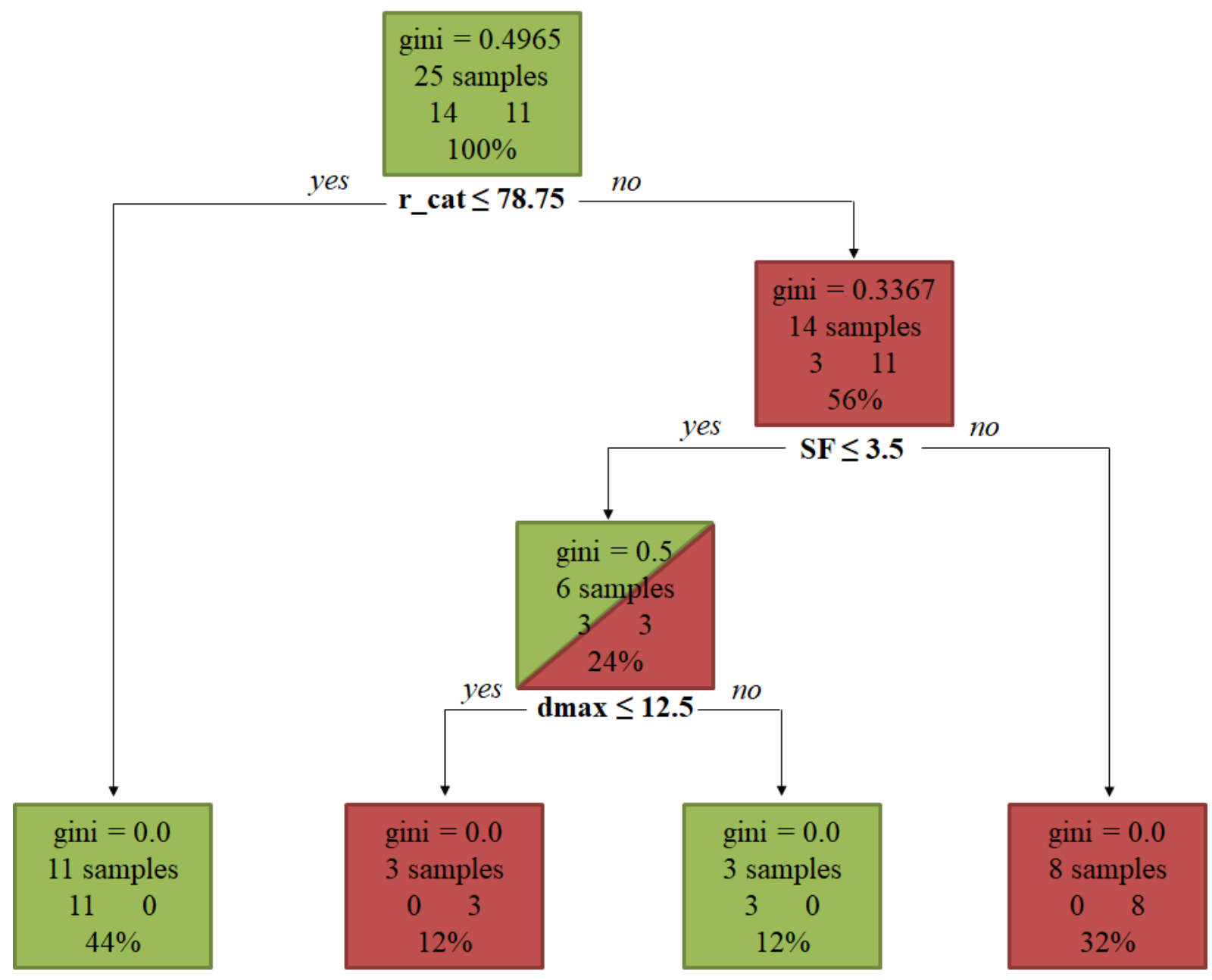




\section{Figure 4}

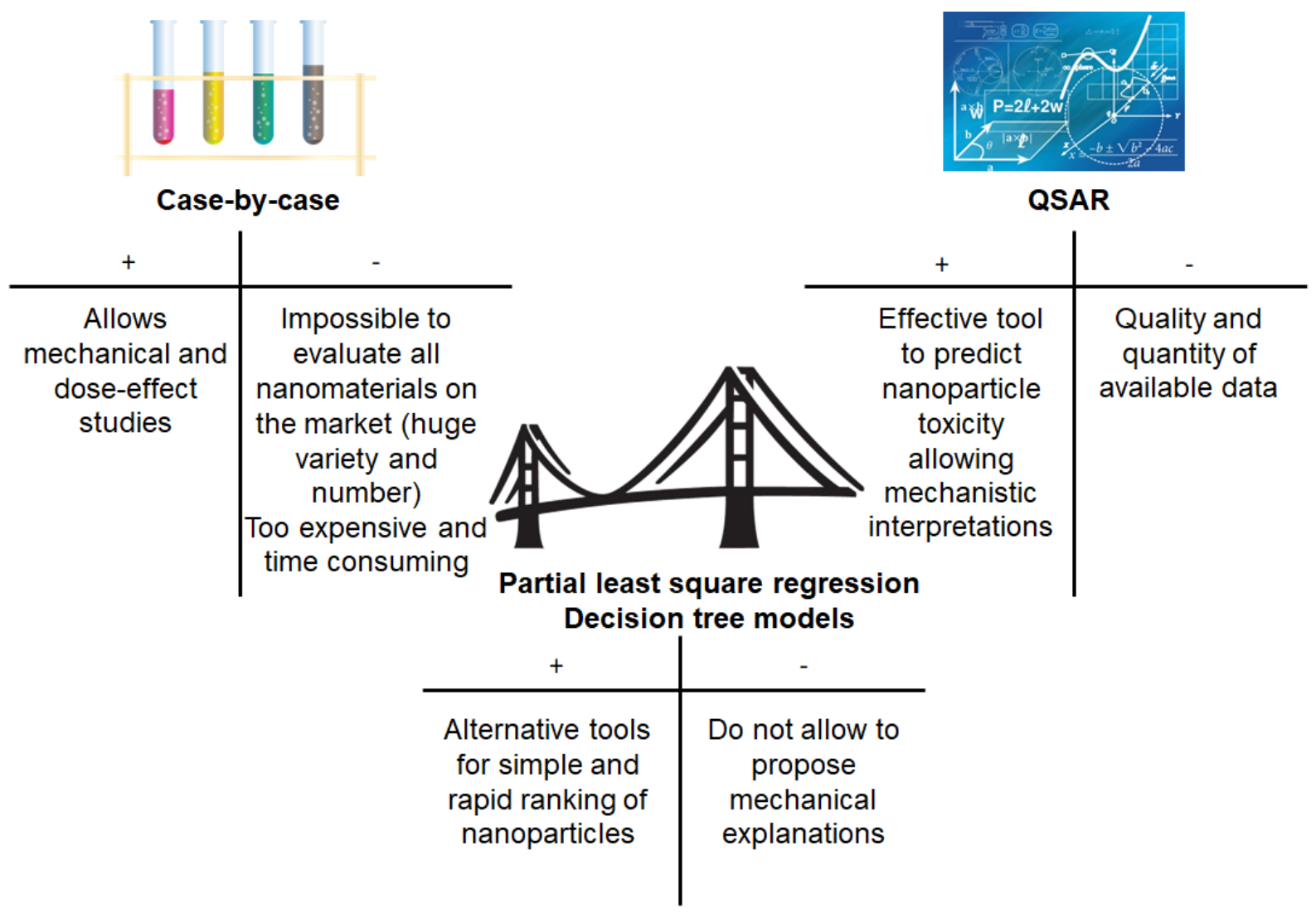




\section{Supplementary information}

Towards an alternative to nano-QSAR for nanoparticle toxicity ranking in case of small datasets

Valérie Forest*a, Jean-François Hochepied ${ }^{\mathrm{b}, \mathrm{c}}$, Lara Leclerc ${ }^{\mathrm{a}}$, Adeline Trouvéb,c, Khalil Abdelkebir $^{\mathrm{b}, \mathrm{c}}$, Gwendoline Sarry ${ }^{\mathrm{a}}$, Vincent Augusto ${ }^{\mathrm{d}}$, Jérémie Pourchez ${ }^{\mathrm{a}}$.

${ }^{\text {a } M i n e s ~ S a i n t-E t i e n n e, ~ U n i v ~ L y o n, ~ U n i v ~ J e a n ~ M o n n e t, ~ I N S E R M, ~ U ~} 1059$ Sainbiose, Centre CIS, F-42023 Saint-Etienne, France.

${ }^{\mathrm{b}}$ Mines ParisTech, PSL Research University, MAT - Centre des matériaux, CNRS UMR 7633, BP 8791003 Evry, France.

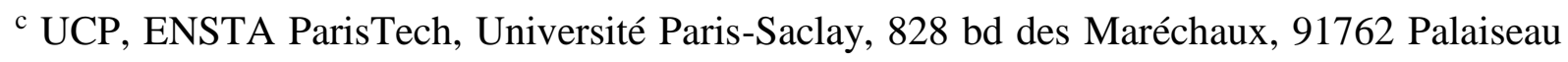
cedex France.

${ }^{\mathrm{d}}$ Mines Saint-Etienne, Univ Clermont Auvergne, CNRS, UMR 6158 LIMOS, Centre CIS, F42023 Saint-Etienne France.

* Corresponding author:

Valérie Forest:

Mines Saint-Etienne, 158 cours Fauriel, CS 62362, 42023 Saint-Etienne Cedex 2. FRANCE.

Email address: vforest@emse.fr - Telephone number: +33477499776

ORCID: 0000-0003-3124-0413

\section{Journal of Nanoparticle Research}


Besides the assessment of cytotoxicity by the LDH release assay, the pro-inflammatory response and the oxidative stress were investigated through the assessment of the TNF- $\alpha$ and reactive oxygen species (ROS) production respectively.

\section{Methods}

As for the LDH assay, the RAW 264.7 cells were seeded in 96-well-plates (100 000 cells in 50 $\mu \mathrm{L}$ of medium per well) and were allowed to adhere overnight. Nanoparticles were diluted in cell culture medium to reach the following final concentrations: $15,30,60$ and $120 \mu \mathrm{g} / \mathrm{mL}$. Nanoparticles were added to cells.

TNF- $\alpha$ production - After a $24 \mathrm{~h}$ incubation with nanoparticles, the production of TNF- $\alpha$ was assessed in the supernatant using a commercial ELISA Kit (Quantikine ${ }^{\circledR}$ Mouse TNF- $\alpha$ Immunoassay; R\&D Systems, Lille, France) according to the manufacturer's instructions. The optical density of each sample was determined using a microplate reader (Multiskan RC; Thermolabsystems, Helsinki, Finland) set to $450 \mathrm{~nm}$. A standard curve was established and results were expressed in picograms of TNF- $\alpha$ per milliliter of supernatant. Three independent experiments were performed, each in quadruplicate and the production of TNF- $\alpha$ was reported to that of control cells (incubated without nanoparticles).

Reactive oxygen species (ROS) production - A large array of ROS activity can be assessed with the OxiSelect ${ }^{\mathrm{TM}}$ ROS Assay Kit (Euromedex, Mundolsheim, France). The assay uses the conversion of a non-fluorescent substrate, 2.7'-dichlorodihydrofluorescein diacetate that can easily diffuse through cell membranes and be converted into a fluorogenic molecule $2^{\prime} .7^{\prime}$ dichlorodihydrofluorescein (DCF) in presence of ROS: fluorescence amount is directly related to ROS level. Fluorescence was detected using a Fluoroskan Ascent fluorometer (Ex: $480 \mathrm{~nm}$, Em: $530 \mathrm{~nm}$, Thermolabsystems) after a $90 \mathrm{~min}$ and a $24 \mathrm{~h}$ incubation of cells with the nanoparticles. A positive control was included incubating cells with $\mathrm{H}_{2} \mathrm{O}_{2}(1 \mathrm{mM})$. Three 
independent experiments were performed, each in quadruplicate and the generation of ROS was reported to that of the negative control (cells incubated without nanoparticles).

Expression of the results: biological scores - As for the LDH assay, means were calculated between the 4 doses to get an average signal and this signal was normalized to the negative control (signal obtained for the cells incubated alone, i.e. without nanoparticles). Therefore, the basal signal obtained for the control was set to 1 .

\section{$\underline{\text { Results }}$}

PLS analysis

\begin{tabular}{|c|c|c|c|}
\hline Sample & TNF- $\alpha$ & ROS 90min & ROS 24h \\
\hline AlOOH-1 & 1.13 & 0.92 & 0.74 \\
\hline AlOOH-2 & 0.96 & 0.89 & 0.69 \\
\hline AlOOH-3 & 1.00 & 0.88 & 0.69 \\
\hline AlOOH-4 & 0.99 & 0.88 & 0.67 \\
\hline AlOOH-5 & 0.94 & 0.92 & 0.76 \\
\hline $\mathbf{N i}(\mathbf{O H})_{2-1}$ & 3.80 & 1.17 & 1.15 \\
\hline $\mathbf{N i}(\mathbf{O H})_{2}-2$ & 2.08 & 1.07 & 1.41 \\
\hline $\mathrm{Ni}(\mathrm{OH})_{2}-3$ & 3.51 & 1.21 & 1.10 \\
\hline $\mathbf{N i}(\mathbf{O H})_{2}-4$ & 2.73 & 1.02 & 1.04 \\
\hline $\mathrm{Ni}(\mathrm{OH})_{2}-5$ & 2.54 & 1.08 & 1.06 \\
\hline $\mathrm{NiO}$ & 1.19 & 1.08 & 2.77 \\
\hline $\mathrm{ZnO}-1$ & 0.83 & 1.14 & 1.40 \\
\hline $\mathrm{ZnO-2}$ & 0.90 & 1.12 & 1.32 \\
\hline $\mathrm{CeO}_{2-1}$ & 1.50 & 1.09 & 1.25 \\
\hline
\end{tabular}




\begin{tabular}{|c|c|c|c|}
$\mathbf{C e O}$ & 1.10 & 1.37 \\
\hline $\mathbf{C e O}_{2}-3$ & 1.68 & 1.08 & 1.36 \\
\hline $\mathbf{C e O}{ }_{2}-4$ & 1.18 & 1.12 & 1.05 \\
\hline $\mathbf{C e O}_{2}-5$ & 1.75 & 1.10 & 1.27 \\
\hline $\mathbf{C e O}_{2}-6$ & 0.93 & 0.91 & 2.29 \\
\hline $\mathbf{T i O}_{2}-1$ & 2.25 & 1.00 & 1.02 \\
\hline $\mathbf{T i O}_{2}-2$ & 0.99 & 0.98 & 1.13 \\
\hline $\mathbf{T i O}_{2}-3$ & 3.58 & 1.02 & 1.04 \\
\hline $\mathbf{T i O}_{2}-4$ & 1.49 & 1.04 & 0.99 \\
\hline $\mathbf{S i O}_{2}-1$ & 2.81 & 1.06 & 1.12 \\
\hline $\mathbf{S i O}_{2}-2$ & 2.66 & 0.97 & 1.01 \\
\hline
\end{tabular}

Suitable predictive models could not be constructed from these data. 